\title{
A novel broadly applicable PCR-RFLP method for rapid identification and subtyping of H58 Salmonella Typhi
}

\author{
Manuela Murgia a, Salvatore Rubino a, b, John Wain c, Rajni Gaind d, Bianca Paglietti a \\ a Department of Biomedical Sciences, University of Sassari, V. le San Pietro 43/B, 07100 Sassari, Italy \\ b Department of Infection and Immunity, King Faisal Specialist Hospital and Research Centre, PO Box 3354 (MBC-03), \\ Riyadh 11211, Saudi Arabia \\ c Norwich Medical School, Norwich Research NRE Park, University of East Anglia, Norwich NR4 7TJ, United Kingdom \\ d Department of Microbiology, Safdarjung Hospital and Assoc VMMC, New Delhi, India
}

\begin{abstract}
Salmonella Typhi (S. Typhi), the human-adapted agent of typhoid fever, is genetically monomorphic. SNPs accumulation divided the $\mathrm{S}$. Typhi population in 85 haplotypes $(\mathrm{H})$ of which one, $\mathrm{H} 58$, has undergone a clonal expansion. The surveillance of $\mathrm{H} 58 \mathrm{~S}$. Typhi is particularly important, especially in areas where typhoid fever is endemic. We developed a simple PCR and PCR-RFLP method to detect and subtype H58 S. Typhi based on the presence of genomic deletion and specific SNPs. The method was validated against $39 \mathrm{~S}$. Typhi isolates of known haplotype, showing $100 \%$ of specificity and high sensitivity, and then used to screen a collection of $99 \mathrm{~S}$. Typhi from Asia, demonstrating a high incidence of H58 S. Typhi in Jordan and India. Our method is designed to be applied in all laboratories with basic molecular biology equipment and few financial resources and allows the surveillance of H58 S. Typhi in resource poor settings.
\end{abstract}

\section{Keywords}

S. Typhi; SNPs; Phylogenetic tree; H58 identification; PCR-RFLP

\section{Introduction}

Salmonella enterica serovar Typhi (S. Typhi) is the human-adapted agent of typhoid fever, a severe and often life-threatening systemic infection transmitted by the faecal-oral route. In 2010 there were an estimated 26.9 million cases of typhoid fever, especially in countries with inadequate sanitation, unsafe water and poor hygiene ( Kariuki et al., 2010, Buckle et al., 2012 and Wain et al., 2015). The fact that $S$. Typhi is restricted to humans has resulted in a very low genetic variability in this serovar, and the genomes of distinct isolates are extremely conserved ( Achtman, 2008 and Holt et al., 2010). For this reason, the most widely used molecular typing methods, including Multilocus Sequence Typing (MLST) and Pulsed-Field Gel Electrophoresis (PFGE), are not sufficiently discriminative for phylogenetic and epidemiological analysis of this pathogen ( Achtman, 2008, Octavia and Lan, 2009 and Thanh et al., 2013). On the contrary, single nucleotide polymorphisms (SNPs) assay has proved to be the most reliable method for S. Typhi genotyping, and over 2000 SNPs were discovered by sequencing different S. Typhi genomes. These SNPs were used to create a phylogenetic tree, which defines $85 \mathrm{~S}$. Typhi haplotypes $(\mathrm{H} 1-\mathrm{H} 85)$, originated through the accumulation of genomic mutations from a common ancestor, the haplotype 45 ( Roumagnac et al., 2006, Achtman, 2008 and Holt et al., 2008). Moreover, insertions and deletions resulting from recombination events were identified in S. Typhi genomes ( Holt et al. 2008). At present, the haplotype $58(\mathrm{H} 58)$ is the most diffused and rapidly expanding among S. Typhi population. The identification of specific SNPs among the $\mathrm{H} 58$ population (haplogroup) further defined two principal lineages (I and II) and different sub-lineages (H58A-H58J, H60-H65) ( Holt et al. 2008). H58 S. Typhi 
strains are endemic in Southeast Asia, India and Africa ( Baltazar et al., 2015 and Wain et al., 2015), and their wide diffusion has been associated to multidrug-resistance (MDR) to the first-line drugs (ampicillin, chloramphenicol, and trimethoprim-sulfamethoxazole) and to reduced susceptibility to the alternative drugs (fluoroquinolones) used in typhoid fever therapy ( Roumagnac et al., 2006, Achtman, 2008, Holt et al., 2010 and Wong et al., 2015). The surveillance of H58 S. Typhi is therefore important, especially in areas where typhoid fever is endemic. Genome sequencing and SNP typing are procedures too expensive and labour-intensive for routine use in most laboratories of molecular biology, even more in low-income countries. For this reason, we have set up a fast and easy PCRRFLP method to detect H58 S. Typhi strains and sub-divided them between linage I and II, based on the presence of specific deletion and SNPs. The method was validated using S. Typhi strains previously sequenced and subjected to SNP typing analysis. We feel that our low-cost assay will be useful in reference and clinical microbiology laboratories in developing countries, where SNP typing is not feasible and PCR-based typing techniques are always more frequently used.

\section{Materials and methods}

\subsection{Identification of $\mathrm{H} 58 \mathrm{~S}$. Typhi by PCR}

In a previous study, genome sequencing of $19 \mathrm{~S}$. Typhi isolates belonging to different haplotypes detected 20 genomic deletions suitable for S. Typhi typing ( Holt et al. 2008). Among these, a deletion of $993 \mathrm{bp}$ affecting STY1507 and STY1508 genes was exclusively present in strains of the H58 haplogroup. Assuming that this deletion could be used to identify H58 S. Typhi strains, we designed primers giving an amplification of $107 \mathrm{bp}$ if the deletion is present (H58 strains), or an amplification of $1100 \mathrm{bp}$ if the deletion is absent (non-H58 strains) (Fig. 1A). The primers were H58F (5'-GCAGGCAAAATCGAAATCAG-3') and H58R (5'-CAAACCGTTGAATCGGAAGT-3'), corresponding respectively to nucleotides 1466515 to 1466534 and 1467614 to 1457595 of S. Typhi CT18 genome (accession number AL513382.1). PCR reactions were carried out in a $25 \mu$ l volume containing $22 \mu \mathrm{l}$ of Platinum ${ }^{\circledR}$ PCR SuperMix (Invitrogen), $1 \mu \mathrm{l}(0.4 \mu \mathrm{M}$ ) of each primer and $1 \mu \mathrm{l}$ (2-10 ng) of DNA. Amplifications were performed on a MJ Research PTC-200 Thermal Cycler with the following conditions: initial denaturation at $94^{\circ} \mathrm{C}$ for $2 \mathrm{~min}$, followed by 30 cycles at $94{ }^{\circ} \mathrm{C}$ for $30 \mathrm{~s}, 60^{\circ} \mathrm{C}$ for 30 $\mathrm{s}, 72{ }^{\circ} \mathrm{C}$ for $1 \mathrm{~min}$, and final extension at $72{ }^{\circ} \mathrm{C}$ for $5 \mathrm{~min}$. PCR products were electrophoresed on $1 \%$ agarose gel and visualized under ultraviolet light after staining with ethidium bromide. 
A

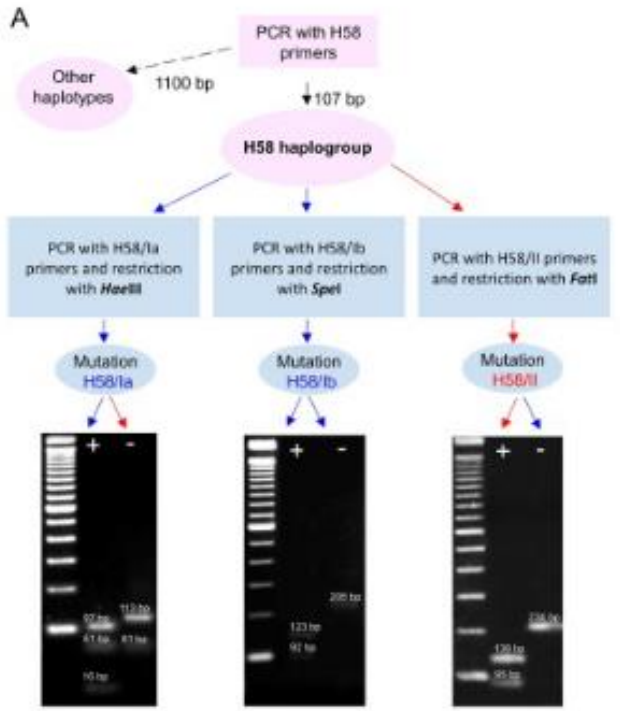

B
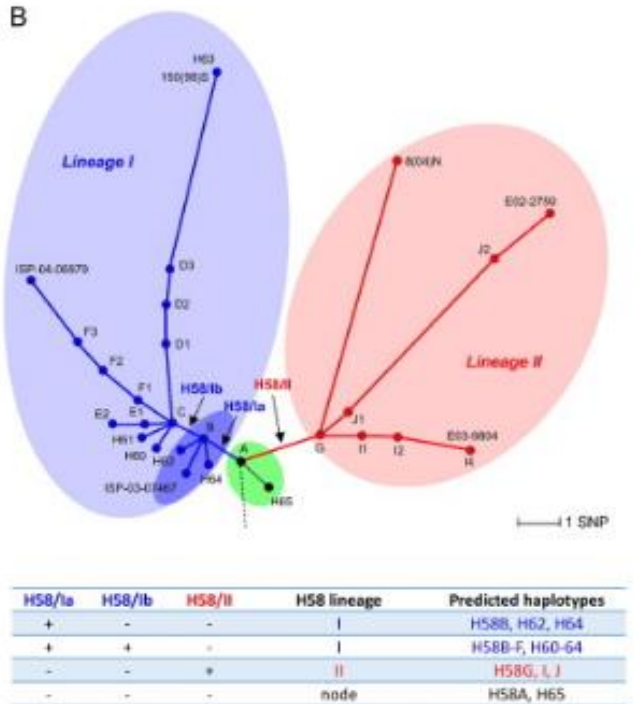

Fig. 1.

Overview of the protocol developed in this study. (A) Schematic representation of the protocol to identify and subdivide H58 S. Typhi strains. (B) Phylogenetic tree of the H58 haplogroup of S. Typhi, adapted from Holt et al. (2011). Letters indic ate the haplotypes $(A=H 58 A, B=H 58 B$, etc. $)$. The positions of the three SNPs investigated in this study are indic ated by arrows, and the groups of haplotypes detected are indic ated by coloured areas. Possible results of the RFLP analysis are summarized in the table.

\subsection{Sub-typing of H58 S. Typhi by PCR-restriction fragment length polymorphism (RFLP)}

The presence of specific SNPs divides the H58 haplogroup in two main lineages and different sublineages (Holt et al. 2010) (Fig. 1B). On this basis, we developed a PCR-RFLP based test. The genome of S. Typhi CT18 was analysed by using the website https://www.neb.com/tools-andresources/interactive-tools/enzyme-finder to find out if restriction sites were presents in correspondence of the mutated nucleotides. Three SNPs suitable for RFLP analysis (here named $\mathrm{H} 58 / \mathrm{la}, \mathrm{H} 58 / \mathrm{lb}, \mathrm{H} 58 / \mathrm{II}$ ) were selected to divide $\mathrm{H} 58 \mathrm{~S}$. Typhi strains in three groups corresponding to lineage I, lineage II and node of the haplogroup, and one sub-group comprising haplotypes H58B, $\mathrm{H} 62$ and $\mathrm{H} 64$ of lineage I ( Fig. 1B). Three set of primers were designed to amplify the regions containing the selected SNPs (Table 1). PCR reactions were carried out as indicated above for H58 primers. Amplification conditions were: initial denaturation at $94{ }^{\circ} \mathrm{C}$ for $2 \mathrm{~min}$, followed by 30 cycles at $94{ }^{\circ} \mathrm{C}$ for $30 \mathrm{~s}, 62{ }^{\circ} \mathrm{C}$ for $30 \mathrm{~s}, 72{ }^{\circ} \mathrm{C}$ for $30 \mathrm{~s}$, and final extension at $72{ }^{\circ} \mathrm{C}$ for $5 \mathrm{~min}$. A total of $10 \mu \mathrm{l}$ of the PCR products were digested in a final volume of $20 \mu \mathrm{l}$ with the corresponding restriction enzyme (Haelll, Invitrogen; Spel, Promega; Fatl, New England Biolabs) ( Table 1) at the conditions indicated by the manufactures. Digested fragments were visualized on $2.5 \%$ agarose gel stained with ethidium bromide (Fig. 1A). 
Table 1

Primer sets, target SNPs, enzymes, and restriction fragments of RFL.P analysis set up in this study.

\begin{tabular}{|c|c|c|c|c|c|c|c|c|}
\hline \multirow[b]{2}{*}{ Primer } & \multirow[b]{2}{*}{ Sequence } & \multirow[b]{2}{*}{ Amplicon size (bp) } & \multicolumn{3}{|l|}{ Target SNPs } & \multicolumn{3}{|l|}{ RFLP } \\
\hline & & & Position in CT18 & Base in $\mathrm{H} 58$ & Base in non-H58 & $\begin{array}{l}\text { Restriction } \\
\text { endonuclease }\end{array}$ & Allele & $\begin{array}{l}\text { Restriction fragments } \\
\text { size (bp) }\end{array}$ \\
\hline H58/la F & AGCGAAGCGTCATTCCAG & 176 & 1193220 & $\mathrm{~T}$ & c & Haell & $T$ & 61,113 \\
\hline $\mathrm{H} 58 / \mathrm{bb} \mathrm{F}$ & ATATCCCGCTACCGGACAGT & 205 & 4798685 & c & $\mathrm{T}$ & Spel & c & 205 \\
\hline $\mathrm{H} 58 / \mathrm{hb} \mathrm{R}$ & TGAGCCTGAAATCCTGAACC & & & & & & $\mathrm{T}$ & 123,82 \\
\hline H58/1IF & AGTTGCTGTTTCTGGCTTCG & 234 & 3694947 & c & G & Fatl & c & 234 \\
\hline H58/M R & CCCCAACCGTAAATATACAAAAGG & & & & & & G & 139,95 \\
\hline
\end{tabular}

\subsection{Validation of the methods}

To preliminary evaluate the specificity of the $\mathrm{H} 58$ primers, we tested 39 control S. Typhi isolates of known haplotype, of which 27 were $\mathrm{H} 58$ and 12 belonged to ten different haplotypes ( Table 2). DNAs of control S. Typhi isolates were kindly supplied by the Wellcome Trust Sanger Institute, Hinxton, UK. The specificity of the PCR-RFLP method in discriminating H58 strains was tested against 12 of the control S. Typhi isolates belonging to lineage I, lineage II and node of H58 ( Table 2). To determine the sensitivity of the assays, DNAs of control strains ( $\mathrm{H} 58$ and non-H58) quantified on a Qubit Fluorometer (Life Technologies) were 2-fold serially diluted to concentrations ranging from 4 to $0.06 \mathrm{ng} / \mu \mathrm{l}$ and used in each PCR reaction.

\begin{tabular}{|c|c|c|c|c|c|c|}
\hline \multirow{2}{*}{ Strain } & \multirow{2}{*}{ Country of isolation } & \multicolumn{3}{|l|}{ SNP typing } & \multicolumn{2}{|l|}{ PCR and RFLP } \\
\hline & & Haplotype by goldengate ${ }^{2}$ & Haplogroup & H58 lineage $^{x}$ & H58 haplogroup by PCR & H58 lineage/predicted haplotype by RFLP \\
\hline CT18 & Vietnam & H1 & H1 & & - & \\
\hline CT114 & Vietnam & $\mathrm{H} 1$ & H1 & & - & \\
\hline$E 00-7866$ & Morocco & H46 & $\mathrm{H} 81$ & & - & \\
\hline E98-3139 & Mexico & HSO & H5Ob & & - & \\
\hline E01-6750 & Senegal & HS2 & H52 & & - & \\
\hline E98-0664 & Kenya & H5S & $\mathrm{H} 50 \mathrm{a}$ & & - & \\
\hline E03-4983 & Indonesia & H59 & $\mathrm{H} 29$ & & - & \\
\hline E02-1687 & Thailand & $\mathrm{H79}$ & H5Oa & & - & \\
\hline M223 & Unknown & $\mathrm{H} 8$ & $\mathrm{H} 50 \mathrm{~b}$ & & - & \\
\hline J185SM & Indonesia & H85 & H29 & & - & \\
\hline JA1254 & Kuwait & $\mathrm{H} 42$ & $\mathrm{H} 42$ & & - & \\
\hline E98-2068 & Bangladesh & $\mathrm{H} 42$ & $\mathrm{H} 42$ & & - & \\
\hline ISP-03-07467 & Moroceo & H58 & H58 & 1 & + & 1/H588, H62, H64 \\
\hline 277466 & UK & H58-B & H58 & 1 & + & 1/H588, H62, H64 \\
\hline JA2827 & Kuwait & H58-B & H58 & i & + & \\
\hline 3T512 & UK & H58-B & H58 & I & + & \\
\hline JA3216 & Kuwait & H58-B & H58 & I & + & \\
\hline JA892 & Kuwait & H58-B & H58 & i & + & \\
\hline ST148 & Jordan & H58-B & H58 & 1 & + & \\
\hline ST 298 & Jordan & H58-B & H58 & i & + & \\
\hline ST 721 & Jordan & H58-B & H58 & i & + & \\
\hline ST 661 & Jordan & H58-B & H58 & 1 & + & 1/H58B, H62, H64 \\
\hline JA 2076 & Kuwait & $\mathrm{H} 62$ & H58 & i & + & 1/H58B, H62, H64 \\
\hline ISP-04-06979 & Central Africa & H58 & H58 & 1 & + & 1/H58C, H58D, H58E, H58F, H6O, H61, H63 \\
\hline CT143 & Vietnam & H58-C & H58 & 1 & + & 1/H58C, H58D, H58E, H58F, H60, H61, H63 \\
\hline DT041 & Vietnam & HSB-C & H58 & i & + & 1/H58C, H58D, HS8E, H58F, H60, H61, H63 \\
\hline AG020 & Vietnam & H58-C & H58 & i & + & \\
\hline AG52 & Vietnam & HSE-C & H58 & i & + & \\
\hline AG118 & Vietnam & H58-C & H58 & i & + & \\
\hline AG169 & Vietnam & H58-C & H58 & 1 & + & \\
\hline AG53 & Vietnam & HS8-E2 & H58 & $i$ & + & \\
\hline $150(98) \mathrm{S}$ & Vietnam & H63 & H58 & i & + & 1/H58C, H58D, H58E, H58F, H60, H61, H63 \\
\hline $8(04) \mathrm{N}$ & Vietnam & H58 & H58 & II & + & H1/H58G, H58I, H58] \\
\hline E02-2759 & India & H58 & $\mathrm{H} 58$ & II & + & H1/H58G, H581, H58j \\
\hline JA2849 & Kuwait & H58-G & H58 & II & + & II/HS8G, H581, H58] \\
\hline E03-9804 & Nepal & H58-14 & H58 & II & + & 11/H58G, H58I, H58] \\
\hline SK6322 & Kenya & H58-j1 & H58 & iI & + & \\
\hline SK6335 & Kenya & H58-J1 & H58 & II & + & \\
\hline JA1050 & Kuwait & H58-A & H58 & Node & + & H58-A o H65 \\
\hline
\end{tabular}

\subsection{S. Typhi isolates tested in this study}

The standardised protocol for $\mathrm{H} 58$ detection and subtyping was applied to screen $99 \mathrm{~S}$. Typhi strains of unknown haplotype, isolated as part of three independent investigations from blood cultures of hospitalized patients suffering typhoid fever in Jordan ( Al-Sanouri et al. 2008), India and Pakistan (unpublished results). Previous characterization of these strains showed that 74 of them (75\%) were MDR (resistant to ampicillin, chloramphenicol, and trimethoprim-sulfamethoxazole), of which 39 were additionally resistant to nalidixic acid (Table 3). 


\begin{tabular}{|c|c|c|c|c|c|c|}
\hline $\begin{array}{l}N^{\circ} \text { of } \\
\text { strains }\end{array}$ & $\begin{array}{l}\text { Country of } \\
\text { isolation }\end{array}$ & $\begin{array}{l}\text { Year of isolation ( } \mathrm{N}^{\circ} \text { of } \\
\text { strains) }\end{array}$ & MDR & $\begin{array}{l}\text { NA- } \\
\text { R }\end{array}$ & $\begin{array}{l}\text { H58 } \\
\text { haplogroup } \\
\text { by PCR }\end{array}$ & $\begin{array}{l}\mathrm{H} 58 \\
\text { lineage/predicted } \\
\text { haplotype by RFLP }\end{array}$ \\
\hline 9 & Jordan & 2004 & - & - & + & $\mathrm{l} / \mathrm{H} 58 \mathrm{~B}, \mathrm{H} 62, \mathrm{H} 64$ \\
\hline 12 & Jordan & $2004(8), 2005(4)$ & + & - & + & $\mathrm{l} / \mathrm{H} 58 \mathrm{~B}, \mathrm{H} 62, \mathrm{H} 64$ \\
\hline 12 & Pakistan & $\begin{array}{l}2000(7), 2001(2), 2002 \\
\text { (3) }\end{array}$ & - & - & - & - \\
\hline 1 & Pakistan & 2000 & - & - & + & I/H58B, H62, H64 \\
\hline 1 & Pakistan & 2000 & + & - & + & I/H58B, H62, H64 \\
\hline 1 & Pakistan & 2000 & + & - & + & $\mathrm{H} 58 \mathrm{~A}, \mathrm{H} 65$ \\
\hline 18 & India & $\begin{array}{l}2004(9), 2006(3), 2008 \\
(4), 2009(2)\end{array}$ & + & - & + & I/H58B, H62, H64 \\
\hline 23 & India & $\begin{array}{l}2004(4), 2006(4), 2007 \\
(4), 2008(10), 2009(3)\end{array}$ & + & + & + & $\mathrm{l} / \mathrm{H} 58 \mathrm{~B}, \mathrm{H} 62, \mathrm{H} 64$ \\
\hline 1 & India & 2008 (1) & - & - & + & $\mathrm{l} / \mathrm{H} 58 \mathrm{~B}, \mathrm{H} 62, \mathrm{H} 64$ \\
\hline 11 & India & $\begin{array}{l}2004 \text { (1), } 2006(1), 2007 \\
(6), 2008(2), 2009(1)\end{array}$ & + & + & + & II/H58G, H58I, H58J \\
\hline 1 & India & 2004 & - & + & + & ॥/H58G, H58I, H58J \\
\hline 1 & India & 2004 & - & - & + & II/H58G, H58I, H58J \\
\hline 3 & India & 2008 (1), 2009 (2) & + & - & + & ॥/H58G, H58I, H58J \\
\hline 1 & India & 2008 & + & + & + & II/H58G, H58I, H58J \\
\hline 1 & India & 2008 & + & + & + & $\mathrm{H} 58 \mathrm{~A}, \mathrm{H} 65$ \\
\hline 1 & India & 2004 & - & - & - & - \\
\hline
\end{tabular}

\section{Results}

\subsection{Validation of methods for identification and subtyping of H58 S. Typhi}

The PCR for H58 S. Typhi identification, conducted on 39 strains of known haplotype, gave an amplification of $107 \mathrm{bp}$ in all the $\mathrm{H} 58$ strains $(n=27)$ and an amplification of $1100 \mathrm{bp}$ in all the haplotypes other than $\mathrm{H} 58(\mathrm{n}=12)$. This confirmed the presence of the deletion of 993 bp only in H58 strains. (Table 2). The detection of the three mutations by PCR-RFLP to subtype H58 strains was tested on 12 strains belonging to lineage I, lineage II and node of H58. The mutation H58/la was correctly detected in all strains of lineage I and not in those of lineage II and node. The second mutation, H58/Ib, was properly identified only in lineage I strains H58C, H63 and ISP-04-06974. Finally, the mutation $\mathrm{H} 58 / \mathrm{II}$ was detected in strains of lineage II and not in those of lineage I and node (Table 2, Fig. 1B). Both methods for identification and subtyping of H58 S. Typhi were thus considered specific at $100 \%$. Moreover, the detection limit of each PCR reaction was $0.06 \mathrm{ng}$ of DNA, indicating the high sensitivity of the developed assays.

\subsection{Analysis of S. Typhi strains of unknown haplotype}

The PCR for H58 identification was used to screen 99 S. Typhi strains of unknown haplotype, showing that $87 \%$ (86/99) of the strains were H58. These included all the isolates from Jordan, $98 \%$ of the isolates from India and $20 \%$ from Pakistan ( Table 3). Subsequently, the H58 strains were subtyped by PCR-RFLP analysis. This revealed that $78 \%(67 / 86)$ of $\mathrm{H} 58$ strains belonged to lineage I, in particular to one of the haplotypes $\mathrm{H} 58 \mathrm{~B}, \mathrm{H} 62$ or $\mathrm{H} 64$, as they carried the mutation $\mathrm{H} 58 / \mathrm{la}$ and not the mutations H58/lb or H58/II (Fig. 1, Table 3). Instead, 20\% (17/86) of H58 strains belonged to lineage II and consequently to one of the haplotypes H58G, H58I or H58J, as they carried only the mutation H58/II. Finally, the remaining $2 \%(2 / 86)$ belonged to the node (H58A) or to H65, as they did not carry any of the tested mutations. H58 S. Typhi from India $(n=62)$, isolated between 2004 and 2009 , were mostly MDR (95\%), and belonged to lineage I (71\%), lineage II (27\%) and node (2\%) of the haplogroup (Table 3). Moreover, $66 \%$ of the MDR H58 Indian strains were also nalidixic acid resistant. S. Typhi from Jordan $(n=21)$, isolated in 2004 and 2005 , were all H58, belonging to lineage 
I, and $57 \%$ of these isolates were MDR. Finally, the three H58 S. Typhi isolated in Pakistan from 2000 to 2002 belonged to lineage I $(n=2)$ and node $(n=1)$ of the haplogroup. Two of these isolates were MDR. On the contrary, all the non-H58 strains $(n=13)$ detected in this study were sensitive to antimicrobials.

\section{Discussion}

The global spread and massive expansion in population size of $\mathrm{S}$. Typhi has been described as being caused by the emergence of the $\mathrm{H} 58$ lineage (Wong et al. 2015). In East Africa specifically, a dramatic increase in typhoid fever has been attributed to H58 (Feasey et al. 2015), but there remains little data on the vast majority of the globe where whole genome sequencing is not available. From the epidemiologic and public health view, being able to detect H58 S. Typhi is essential if we are to understand its global spread and to manage the associated risks ( Wong et al. 2015).

In this study, we have developed a simple, sensitive and easy to use PCR and PCR-RFLP based typing method, which allows the identification and sub-division of the H58 S. Typhi population. Our results showed $100 \%$ specificity. In fact, our PCR successfully detected the presence of the selected deletion of $993 \mathrm{bp}$ in all H58 S. Typhi strains tested, and its absence in all non-H58 strains. We thus confirmed our initial hypothesis, affirming that this deletion is tightly conserved in H58 S. Typhi and it is suitable for an accurate identification of this haplogroup. The same deletion has been included along with other genomic markers in a more complex test for S. Typhi subtyping based on Multiplex LigationDependent Probe Amplification (MLPA) ( Thanh et al. 2013). However, the MLPA method requires specific equipment and is technically demanding and expensive for everyday laboratory practice. Instead, our inexpensive and simple protocol can be useful for preliminary screening of S. Typhi isolates, rapidly detecting the $\mathrm{H} 58$ strains for phylogenetic purpose. Moreover, faster reporting of microbiology results in all clinical microbiology laboratory can significantly decrease the length of hospitalization and the related costs. In this view, we can consider an implementation of our developed PCR method for detecting H58 S. Typhi directly from stool or blood samples without the need for culture, further reducing the time of identification.

Moreover, we developed a PCR-RFLP method, based on the presence of specific SNPs, to sub-divide the $\mathrm{H} 58$ strains into three groups, which will help to follow the circulations of the H58 population. A recent study evidenced a phylogeographical distribution of the H58 S. Typhi, highlighting the importance of discriminate among this population ( Wong et al. 2015). When we applied the protocol to a collection of $99 \mathrm{~S}$. Typhi from India, Pakistan and Jordan, we detected a high percentage of $\mathrm{H} 58$, confirming the diffusion of this haplogroup, particularly in India and Jordan, according with other reports ( Kariuki et al., 2010, Emary et al., 2012, Thanh et al., 2013, Feasey et al., 2015 and Wong et al., 2015). Moreover, we determined that all the H58 Jordan isolates and most of the Indian and Pakistani belonged to H58B, H62 or H64 (lineage I), whereas H58G, H58I or H58J strains (lineage II) were detected only among Indian isolates. Finally, H58A (node) or H65 strains were less common and were not represented in Jordan isolates. These findings are in agreement with other studies based on SNP-typing ( Holt et al., 2012 and Wong et al., 2015), underling the usefulness of our protocol. 
Further, we found that $84 \%$ of $\mathrm{H} 58$ strains in our collection were MDR and $45 \%$ displayed nalidixic acid resistance, whereas all the non-H58 S. Typhi were antibiotic-susceptible. This confirms the association of H58 S. Typhi with high levels of MDR and reduced susceptibility to fluoroquinolones ( Thanh et al., 2013, Wain et al., 2015 and Wong et al., 2015).

In conclusion, we have developed an efficient, low-cost and easy to perform protocol for identifying and sub-dividing the dominant haplogroup of S. Typhi, H58, which has the advantage of being applied in all laboratories with basic molecular biology equipment and few financial resources. We believe that reference laboratories, especially in developing countries, will be able to use this methodology to track the further spread of $\mathrm{H} 58$ and so help to predict the geographical regions at risk in a probabilistic manor.

\section{Acknowledgments}

We wish to acknowledge Kathryn E. Holt and Duy Pham Thanh for supply DNAs of the S. Typhi strains used to validate this study. We also thank Gabriele Carenti for his technical assistance. MM was supported by "Regione Autonoma della Sardegna" through a research Fellowship on fundings PO Sardegna FSE 2007-2013, L.R.7/2007, Promozione Della Ricerca Scientifica e dell'Innovazione Tecnologica in Sardegna. BP is supported by Sardinian Region [POR-FSE 2007-2013-Obiettivo Competitività regionale e occupazione - Asse IV capitale umano - Linea di attività 1.3.1].

\section{References}

Achtman, 2008 M. Achtman: Evolution, population structure, and phylogeography of genetically monomorphic bacterial pathogens (Annu. Rev. Microbiol., 62 (2008), pp. 53-70 http://dx.doi.org/10.1146/annurev.micro.62.081307.162832)

Al-Sanouri et al., 2008 T.M. Al-Sanouri, B. Paglietti, A. Haddadin, M. Murgia, D. Bacciu, M. Youssef, et al: Emergence of plasmid-mediated multidrug resistance in epidemic and non-epidemic strains of Salmonella enterica serotype Typhi (from Jordan J. Infect. Dev. Ctries, 2 (4) (2008), pp. 295-301 http://dx.doi.org/10.3855/jidc.225)

Baltazar et al., 2015 M. Baltazar, A. Ngandjio, K.E. Holt, E. Lepillet, M. Pardos, D. Gandara, et al. Salmonella enterica (Emerg. Infect. Dis., 21 (4) (2015), pp. 655-659

http://dx.doi.org/10.3201/eid2104.141355)

Buckle et al., 2012 G.C. Buckle, C.L.F. Walker, R.E. Black Typhoid fever and paratyphoid fever: systematic review to estimate global morbidity and mortality for 2010 (J. Glob. Health, 2 (1) (2012), p. 10401 http://dx.doi.org/10.7189/jogh.02.010401)

Emary et al., 2012 K. Emary, C.E. Moore, N. Chanpheaktra, K.P. An, K. Chheng, S. Sona, et al. Enteric fever in Cambodian children is dominated by multidrug-resistant $\mathrm{H} 58$ Salmonella enterica serovar Typhi with intermediate susceptibility to ciprofloxacin (Trans. R. Soc. Trop. Med. Hyg., 106 (12) (2012), pp. 718-724 http://dx.doi.org/10.1016/j.trstmh.2012.08.007)

Feasey et al., 2015 N.A. Feasey, K. Gaskell, V. Wong, C. Msefula, G. Selemani, Kumwenda, et al. Rapid emergence of multidrug resistant, H58-lineage Salmonella Typhi in Blantyre, Malawi (PLoS Negl. Trop. Dis., 9 (4) (2015), Article e0003748 http://dx.doi.org/10.1371/journal.pntd.0003748) 
Holt et al., 2008 K.E. Holt, J. Parkhill, C.J. Mazzoni, P. Roumagnac, F.X. Weill, I. Goodhead, et al. Highthroughput sequencing provides insights into genome variation and evolution in Salmonella Typhi (Nat. Genet., 40 (8) (2008), pp. 987-993 http://dx.doi.org/10.1038/ng.195)

Holt et al., 2010 K.E. Holt, S. Baker, S. Dongol, B. Basnyat, N. Adhikari, S. Thorson, et al. Highthroughput bacterial SNP typing identifies distinct clusters of Salmonella Typhi causing typhoid in Nepalese children (BMC Infect. Dis., 10 (2010), p. 144 http://dx.doi.org/10.1186/1471-2334-10-144)

Holt et al., 2011 K.E. Holt, M.D. Phan, S. Baker, P.T. Duy, T.V.T. Nga, S. Nair, et al. Emergence of a globally dominant inchi1 plasmid type associated with multiple drug resistant typhoid (PLoS Negl. Trop. Dis., 5 (7) (2011) http://dx.doi.org/10.1371/journal.pntd.0001245)

Holt et al., 2012 K.E. Holt, S. Dutta, B. Manna, S.K. Bhattacharya, B. Bhaduri, D.J. Pickard, et al. Highresolution genotyping of the endemic Salmonella Typhi population during a Vi (Typhoid) vaccination trial in Kolkata (PLoS Negl. Trop. Dis., 6 (1) (2012), Article e1490 http://dx.doi.org/10.1371/journal.pntd.0001490)

Kariuki et al., 2010 S. Kariuki, G. Revathi, J. Kiiru, D.M. Mengo, J. Mwituria, J. Muyodi, et al. Typhoid in Kenya is associated with a dominant multidrug-resistant Salmonella enterica serovar Typhi haplotype that is also widespread in Southeast Asia (J. Clin. Microbiol., 48 (6) (2010), pp. 2171-2176 http://dx.doi.org/10.1128/JCM.01983-09)

Octavia and Lan, 2009 S. Octavia, R. Lan Multiple-locus variable-number tandem-repeat analysis of Salmonella enterica serovar Typhi (J. Clin. Microbiol., 47 (8) (2009), pp. 2369-2376 http://dx.doi.org/10.1128/JCM.00720-07)

Roumagnac et al., 2006 P. Roumagnac, F.X. Weill, C. Dolecek, S. Baker, S. Brisse, N.T. Chinh, et al. Evolutionary history of Salmonella Typhi (Science, 314 (5803) (2006), pp. 1301-1304 http://dx.doi.org/10.1126/science.1134933)

Thanh et al., 2013 D.P. Thanh, N.T.V. Thieu, C.T. Thuy, M. Lodén, K. Tuin, J.I. Campbell, et al. Identification of Salmonella enterica serovar Typhi genotypes by use of rapid multiplex ligationdependent probe amplification (J. Clin. Microbiol., 51 (9) (2013), pp. 2950-2958 http://dx.doi.org/10.1128/JCM.01010-13)

Wain et al., 2015 J. Wain, R.S. Hendriksen, M.L. Mikoleit, K.H. Keddy, R.L. Ochiai Typhoid fever (Lancet, 385 (9973) (2015), pp. 1136-1145 http://dx.doi.org/10.1016/S0140-6736(13)62708-7)

Wong et al., 2015 V.K. Wong, S. Baker, D.J. Pickard, J. Parkhill, A.J. Page, N.A. Feasey, et al. Phylogeographical analysis of the dominant multidrug-resistant $\mathrm{H} 58$ clade of Salmonella Typhi identifies inter- and intracontinental transmission events (Nat. Genet., 47 (6) (2015), pp. 632-639 http://dx.doi.org/10.1038/ng.3281) 